\title{
A finite element analysis of the push-out test: Influence of test conditions
}

\author{
W. J. A. Dhert, C.C. P. M. Verheyen, ${ }^{*, \ddagger}$ L. H. Braak, J. R. de Wijn; C. P. A.T. Klein, \\ K. de Groot; and P. M. Rozing ${ }^{+}$ \\ *Biomaterials Research Group, Department of Biomaterials, School of Medicine, University of \\ Leiden; ${ }^{\dagger}$ Biomaterials Research Group, Department of Orthopaedic Surgery, University Hospital \\ Leiden; ${ }^{8}$ Eindhoven University of Technology, Faculty of Mechanical Engineering, Eindhoven, \\ The Netherlands
}

The commonly used method for quantitative evaluation of the strength of a boneimplant interface is the push-out test. In order to give an impulse to standardization and to gain more insight in the biomechanics of the push-out test, a finite element analysis of this test was performed. This study focused on the influence of test conditions on the push-out results. The influence of the following four parameters on the interface stress distribution was tested: (a) clearance of the hole in the support jig, (b) Young's modulus of the implant; (c) cortical thickness; and (d) implant diameter. The distance between the implant and the support jig turned out to be very critical for the occurrence of peak stresses in the interface. Variations of the Young's modulus of the implants resulted in a wide range of interface shear stresses. Variation of the cortical thickness showed a reciprocal relationship between cortical thickness and interface shear stress. However, the interface stress distribution remained uniform under the specific test circumstances. These findings also hold for variations in implant diameter. The present investigation shows that the clearance of the hole in the support jig, and the Young's modulus of the implant are parameters which most strongly influence the interface stress distribution. The clearance of the hole in the support jig is the most critical parameter, but also the parameter that can be controlled most easily. Lack of standardization with regard to these parameters can lead to uninterpretable test results. It is recommended that the clearance of the hole in the support jig is at least $0.7 \mathrm{~mm}$ and that push-out results are only compared with each other when materials with similar Young's modulus are concerned.

\section{INTRODUCTION}

The biocompatibility of an orthopedic or dental implant can be investigated by the use of various methods among which testing the strength of the healed implant-bone interface by the push-out test is used frequently. ${ }^{1-15}$ Since this push-out model is used widely, comparisons of test results between different materials and centers are very interesting. In Table I, a survey is

${ }^{\ddagger}$ To whom correspondence should be addressed at Department of Biomaterials, Building 55, School of Medicine, University of Leiden, Rijnsburgerweg 10, 2333 AA Leiden, The Netherlands.

Journal of Biomedical Materials Research, Vol. 26, 119-130 (1992)

(C) 1992 John Wiley \& Sons, Inc.

CCC 0021-9304/92/010119-12\$4.00 


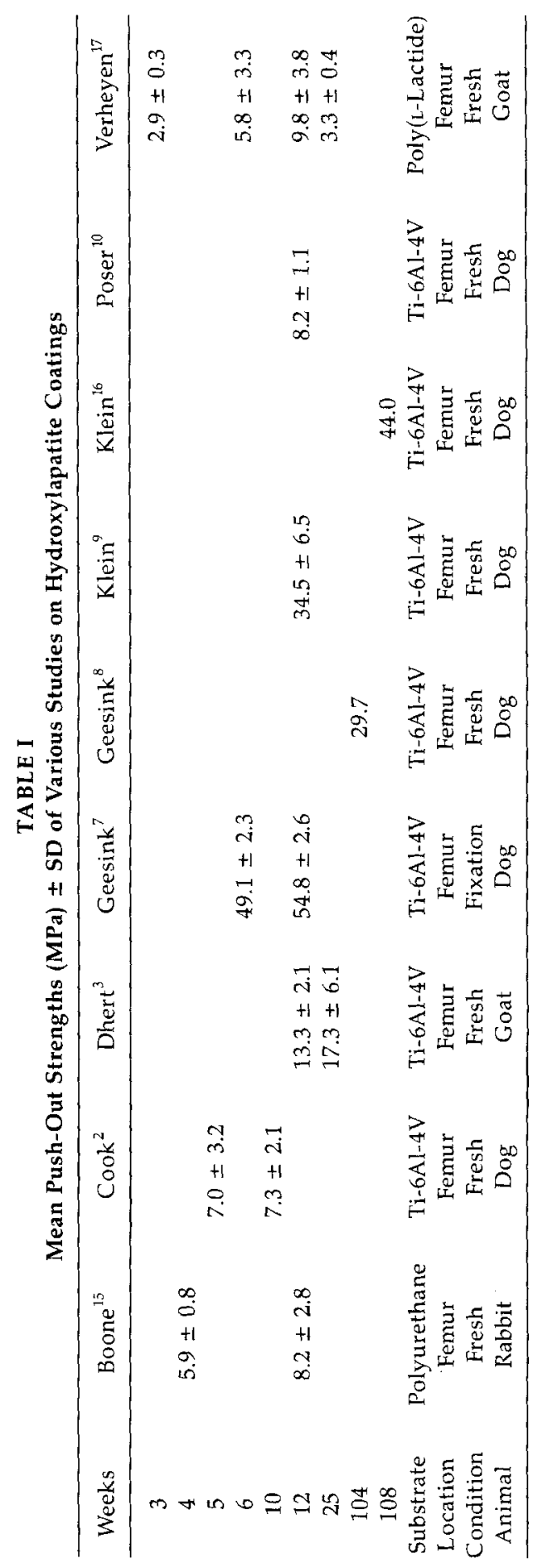


given of various push-out results on hydroxylapatite coatings with approximately equal surface characteristics. ${ }^{2,3,7-10,15-17}$ There exists a wide variety of results that makes comparisons between these studies difficult. For instance, at 12 weeks follow-up, mean push-out strengths are reported between 8.2 for freshly tested specimens, ${ }^{10,15}$ and $54.8 \mathrm{MPa}$ for formalin-fixed specimens. ${ }^{7}$ This large difference might be partially due to the method of specimen preparation (fresh versus formalin fixation), but in another study Klein ${ }^{9}$ reported a push-out strength of $34.5 \mathrm{MPa}$ for freshly tested specimens. Thus, with identical specimen preparation, there still exists a large scatter in data. It was already stated by Black ${ }^{18}$ that when a push-out test is used, the test conditions need to be described thoroughly. Furthermore, it is necessary to gain more insight into the biomechanical characteristics of the push-out model. In this study a finite element analysis of the push-out test was performed in which the effect of frequently varying parameters (plug-hole clearance, Young's modulus of implant, cortical thickness, and implant diameter) on the interfacial shear strength was calculated.

The analysis done was a linear one and only a first step toward a complex analysis in which damage mechanics and nonlinear material behavior have to be incorporated to predict the failure process during a push-out test. In this analysis the three-dimensional state of stress is important, but also the criteria by which this state of stress is evaluated. In our analysis we compare the average shear stress calculated in a classical manner with the shear stresses calculated in a finite element model. The linear elastic behavior of this model gives insight into the regions of high stresses and probably these regions are starting points for cracks; but crack forming or other damage phenomena were not the point of interest in our analysis.

\section{METHODS}

The finite element model was based on the "single cortical push-out model" which is characterized by implantation of a cylinder in cortical bone. This model has the advantage of being generally applicable in the testing of implant fixation in diaphyseal cortical bone of any bone type (for instance, femur, humerus, or tibia) or species, and is used as a standard model for the push-out test in our group. ${ }^{3,4,7-9,14}$ In general, this test has the same biomechanical characteristics as the push-out tests used by other groups. ${ }^{1,11}$ After a certain period of time, the test animals are sacrificed, and a semicircular piece of bone containing the implant is sawn out and placed on a support jig (Fig. 1). Periosteal bone overgrowth was neglected in the finite element model since in practice we remove this bone by polishing. ${ }^{3}$ The implant is pushed out from the surrounding bone. The peak-force that results in movement of the implant is considered as the push-out force. The contact area of bone to implant is calculated or estimated from the implant diameter and the thickness of the bone surrounding the implant. The "interfacial shear strength" is calculated using the following formula:

$$
S=\frac{F}{\pi \times d \times t}
$$




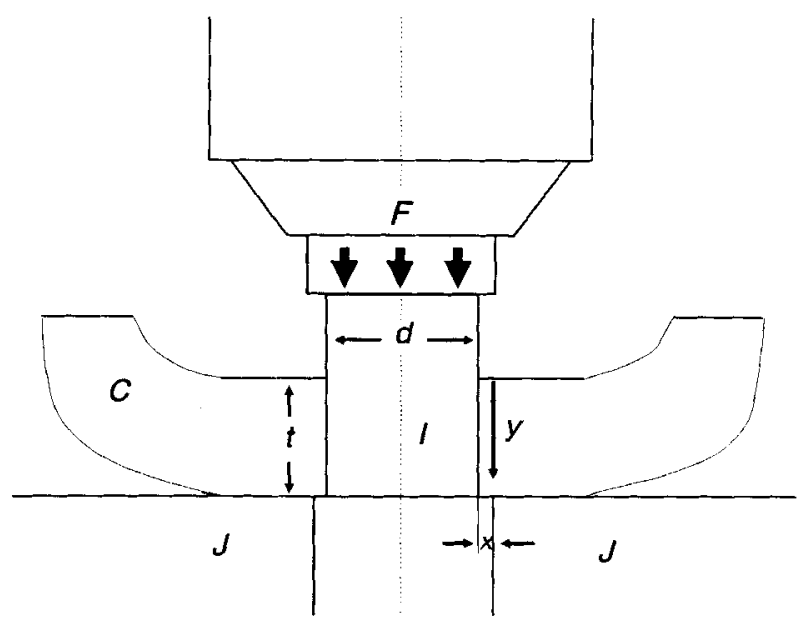

Figure 1. Schematic drawing of the push-out test. $F=$ force applied on implant; $\mathrm{I}=$ implant; $\mathrm{C}=$ cortex; $\mathrm{J}=$ support jig; $y=$ coordinate along the interface. Geometrical parameters, varied in the analysis: $x=$ Clearance of hole in support jig, $d=$ implant diameter, $t=$ cortical thickness.

where $S$ represents the interface shear strength $(\mathrm{MPa}), F$ the push-out force $(\mathrm{N}), d$ the plugdiameter $(\mathrm{mm})$, and $\bar{t}$ the average cortical thickness (mm).

In our model the push-out test can be considered to be axisymmetric, and a two-dimensional finite element model was developed, using quadrangular ring elements. The grid-composition and element distribution are shown in Figure 2. The final density of the element mesh was determined by incremental mesh refinements in the regions of the highest stresses. In this model, the cortical bone was considered to be homogeneous, linear elastic, and isotropic, with a Young's modulus of $15 \mathrm{GPa}$, and a Poisson ratio of 0.3 . At the interface between bone and implant continuity in the displacements was assumed. Between the support jig and the bone only vertical forces were working, and

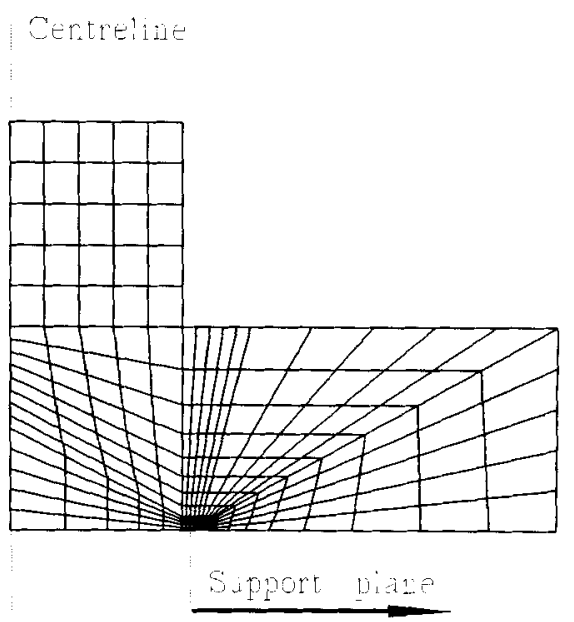

Figure 2. Graphical representation of the two-dimensional finite element model showing grid-composition and element distribution. 
friction at this interface was neglected. The stiffness of the support jig was considered to be infinite high. An axial load of $100 \mathrm{~N}$ was applied from the medullar site on the implant. Based upon practical considerations, the following values were chosen for the initial test parameters:
$x$ : Clearance of hole in support jig $\quad=0.7 \mathrm{~mm}$
$E$ : Implant Young's modulus $(\mathrm{Ti}-6 \mathrm{Al}-4 \mathrm{~V})=110 \mathrm{GPa}$
$t$ : Cortical thickness $\quad=3 \mathrm{~mm}$
$d$ : Implant diameter $\quad=5 \mathrm{~mm}$

In the next step, one of the parameters $x, E, t$, and $d$ was varied according to the following schedule, while the other three parameters were kept at their initial value:

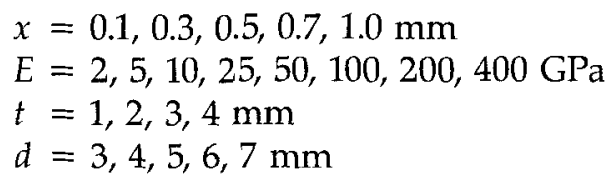

The parameter values chosen in this study cover most test set-ups which are reported in literature.

For each single combination of $x, E, t$, and $d$, the principal stresses in the first layer of elements in the bone along the bone-implant interface were calculated. From these data the interfacial shear stress was determined. A comparison was made between the situation where the parameters $x, E, t$, and $d$ have ideal values, and situations where these parameters have more extreme values resulting in an unequal interface stress distribution. All calculations were performed on a personal computer, using the GIFTS* finite element analysis package.

\section{RESULTS}

\section{Clearance of hole in support jig $(x)$}

Varying $x$ results in the interface stress patterns as shown in Figure 3 . It is obvious that the distance between implant and support jig influences the interface stress considerable. A small oversize of the hole in the support jig, represented by a value for $x$ of $0.1 \mathrm{~mm}$, results in high stresses at the site where the jig edge supports the bone (lateral). When $x$ is increased, the interface stress distribution becomes more uniform.

\section{Implant Young's modulus $(E)$}

In Figure 4, the interface stress patterns are shown for $E$ varying between 2 and $400 \mathrm{GPa}$. The interface stress distribution shows to be most uniform for implants with a Young's modulus of approximately $50 \mathrm{GPa}$. At lower Young's

${ }^{*}$ Version 4.3, CASA/GIFTS Inc., Tucson, AZ. GIFTS is a general finite element analysis package that can be used for solving linear problems in the field of statics, dynamics, and thermoanalysis. Pre- and postprocessing facilities are available. 


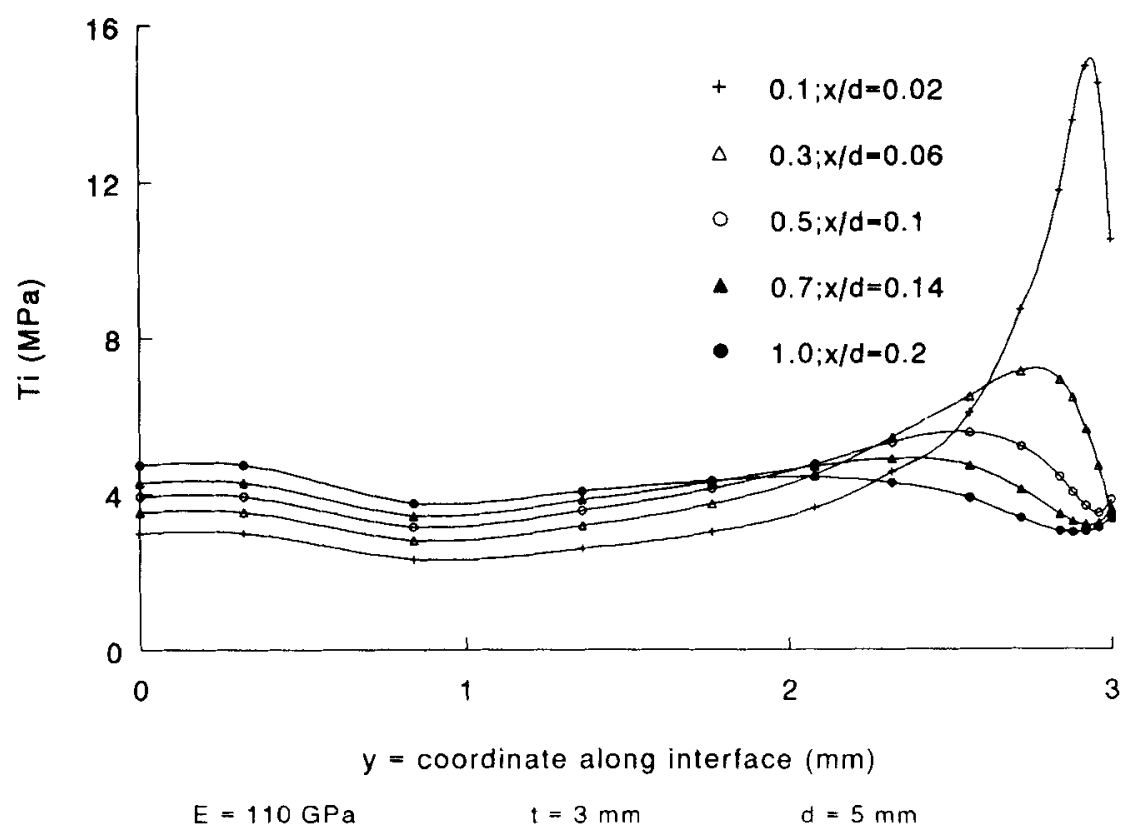

Figure 3. Interface stress distributions for implant-support jig distances $(x)$ varying between $0.1 \mathrm{~mm}$ and $1.0 \mathrm{~mm} . \mathrm{Ti}=$ interface stress in MPa. Legend indicates $x$ in $\mathrm{mm}$.

moduli, the interface stress distribution becomes less uniform, and the higher stress values are located at the site from which the load is applied on the implant (medial). Increasing $E$ does not affect the interface stress distribution considerable, although the interface stress will be a little higher at the site where the jig edge supports the bone (lateral).

\section{Cortical thickness $(t)$}

In Figure 5, the interface stress patterns are shown for $t$ varying from 1 to $4 \mathrm{~mm}$. The stresses are represented as a function of the relative position along the cortex (percentage of cortex). A decrease in $t$ results in a progressive increase of the interface shear stress, but without strongly affecting the stress distribution. This increase is explained by the reciprocal relationship between shear stress and cortical thickness at constant force.

\section{Implant diameter $(d)$}

Varying $d$ from 3 to $7 \mathrm{~mm}$ revealed that the interface stress distribution is hardly affected by this, both with respect to the shape and to the position of the curves. Therefore, it is stated that $d$ has not a significant influence on the interface stress distribution.

In Figure 6, the interface stress patterns are shown for the reference situation (curve 1) with a relative equal stress distribution, and a situation where 


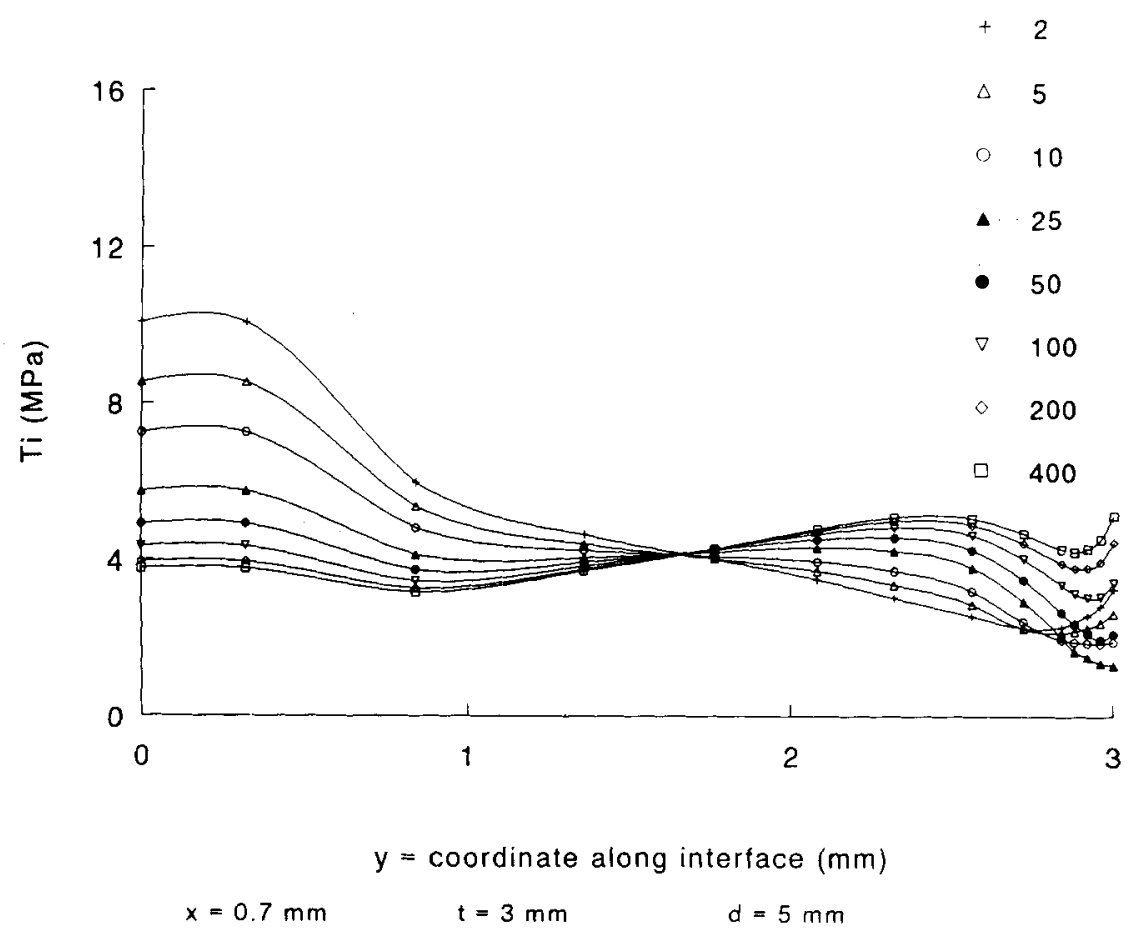

Figure 4. Interface stress distributions for the implant Young's Moduli $(E)$ varying between 2 and $400 \mathrm{GPa}$. Ti = interface stress in MPa. Legend indicates $E$ in $\mathrm{GPa}$.

the stress distribution is very unequal (curve 2) due to variation of both the parameters $x$ and $E$.

\section{DISCUSSION}

In the present investigation some practical biomechanical aspects of the push-out test have been evaluated. Comparing push-out results of various investigators is very interesting to get a proper evaluation of the fixation in bone of a material. It was discussed recently that test circumstances can substantially influence the push-out results. ${ }^{19,20}$ Black $^{18}$ published a survey of factors that can influence test results and which need to be standardized or at least mentioned. In the present study the authors aimed at the biomechanics of the practical situation to gain more insight in the test itself, and in the comparability and reliability of results from different push-out tests.

The authors chose the following parameters as being most relevant: (a) clearance of the hole in the support jig, (b) Young's modulus of the implant, (c) cortical thickness, and (d) implant diameter.

With respect to the clearance of the hole in the support jig, it was shown that a small oversize of the hole results in high stresses at the site where the jig edge supports the bone. A clearance of more than $0.7 \mathrm{~mm}$ decreases this peak to a minimum. Most of the tests described in the literature do not pay any attention to this critical factor, or surprisingly suggest a tight clearance 


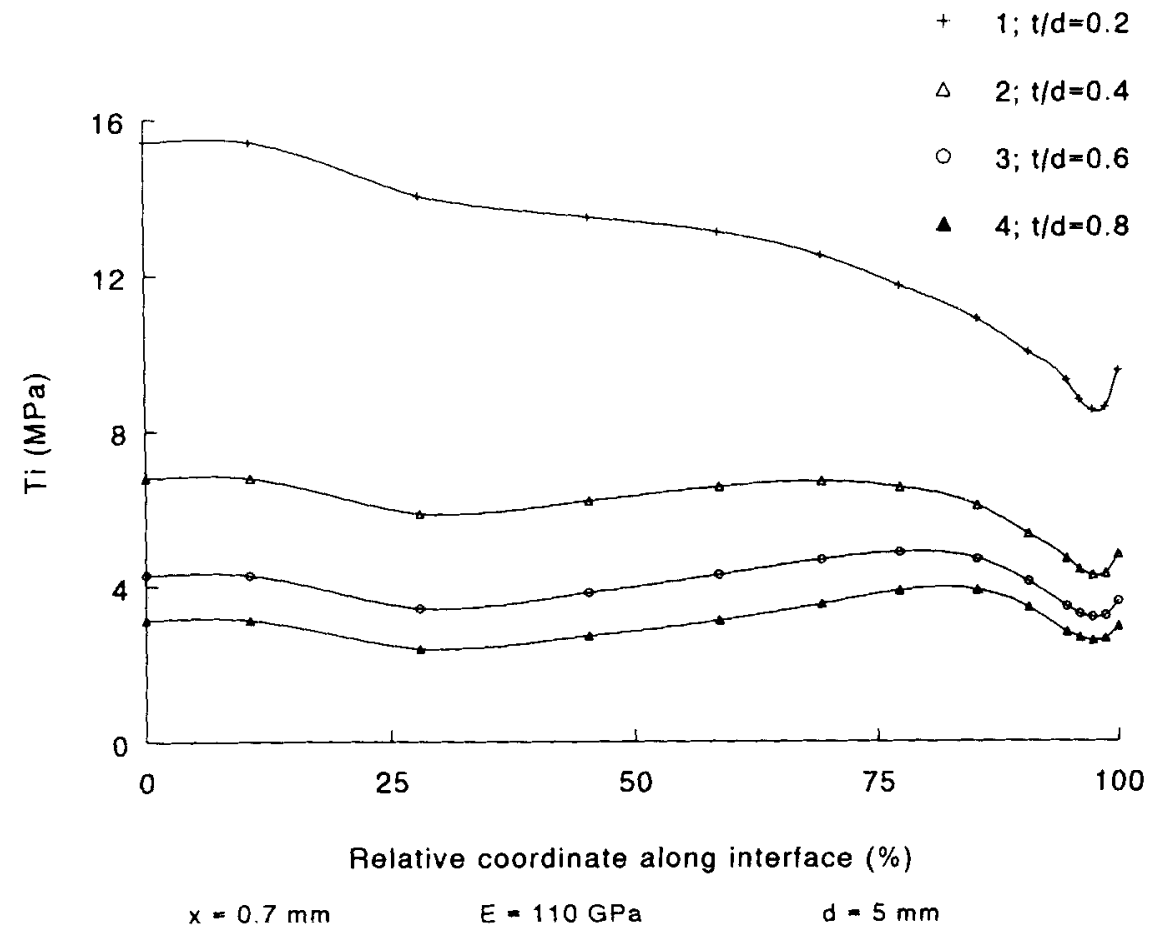

Figure 5. Interface stress distributions for the cortical thickness $(t)$ varying from 1 to $4 \mathrm{~mm}$. $T i=$ interface stress in $\mathrm{MPa}$. Stresses are here represented as a function of the relative position along the cortex from $0 \%$ (inner site of cortex) to $100 \%$ (outer site with support jig). Legend indicates $t$ in $\mathrm{mm}$.

to be ideal. This is not the case, on the contrary, when the clearance is for instance $0.1 \mathrm{~mm}$, the resulting unequal stress distribution gives test results that are difficult to evaluate and that do not reflect the actual ultimate shear stress. Comparing the stress distributions for a clearance of $0.1 \mathrm{~mm}$ and $1.0 \mathrm{~mm}$, there occurs at a constant load of $100 \mathrm{~N}$ for $x=0.1 \mathrm{~mm}$, a stress peak which is almost four times higher than the maximum stress calculated for $x=1.0 \mathrm{~mm}$ (see Fig. 3). Thus, the clearance is a critical parameter, but can be controlled easily. Shiradzi-Adl ${ }^{21}$ recently stated that to get a uniform stress distribution along the interface, it is theoretically required not to support the sample under the implant, but to support the bone around the implant. Our study confirms these results partially, however with regards to the practical execution of a push-out test, we suggest that it is still easier to support the sample using a jig with an adequately oversized hole.

Varying the Young's modulus of the implant revealed again that the interface stress distribution is in most situations not uniform. A low Young's modulus results in considerable higher stresses at the medial site of the cortex, from which the load is applied on the implant. A high Young's modulus results in slightly higher stresses at the lateral site of the cortex where the jig edge supports the bone. But in the latter situation the interface stress distribution is much more uniform compared to the situation of a low Young's modulus of 


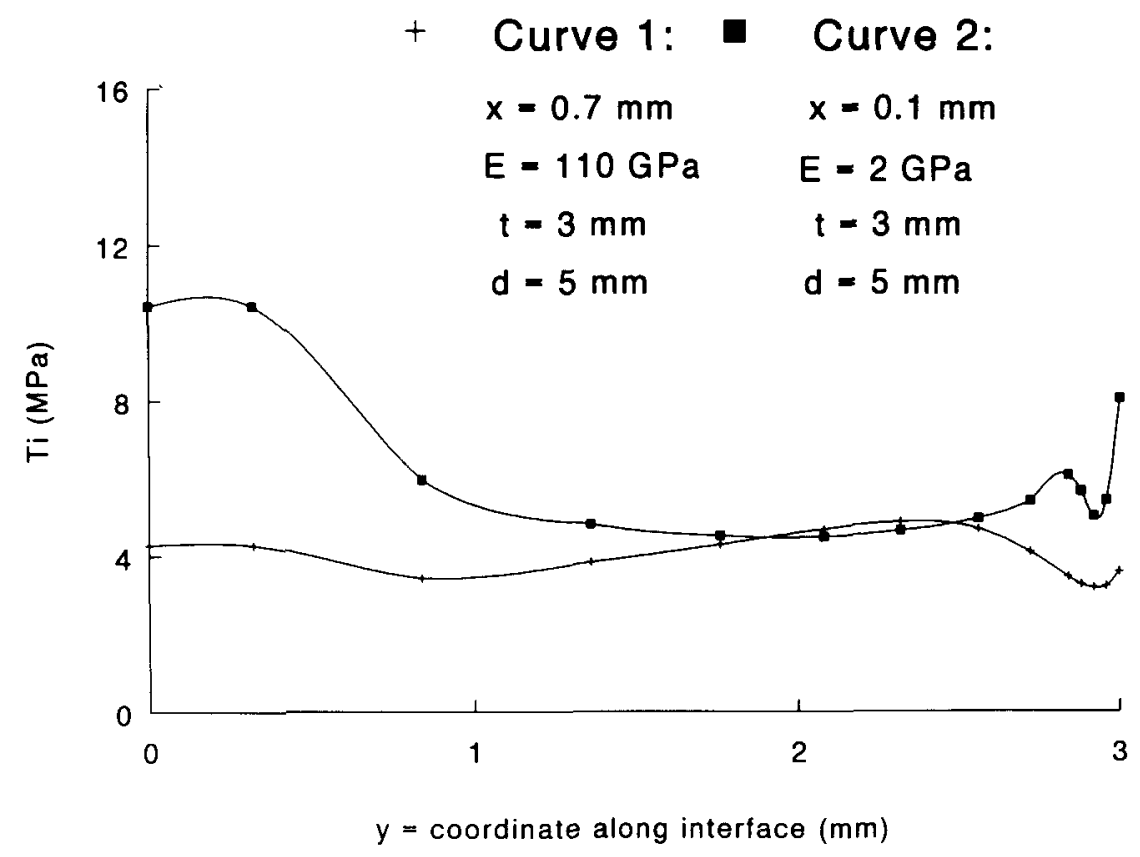

Figure 6. Interface stress distributions for two situations that can occur in practice. Curve 1 shows the reference situation, and curve 2 shows a situation where parameters are changed in unfavorable values.

the implant. These findings show that the strength of the bone-implant interface of materials with different Young's moduli is uninterpretable by pushout test. In most situations, the Young's modulus of the implant cannot be standardized or influenced, since the major goal of a push-out test is the evaluation of materials that might vary with respect to their Young's moduli. The relevance of push-out testing of a material with a low Young's modulus is therefore limited to comparing different follow-up periods or to a comparison with other materials having the same Young's modulus.

The cortical thickness and implant diameter did hardly influence the interface stress distribution. The actual stress values did change when varying the cortical thickness, but the uniformity of the stresses along the interface did not change considerable. It is suggested that in the situations described in this study, the influence of both the cortical thickness as well as the implant diameter on the interfacial stress distribution can be neglected. Thus, when the other parameters are standardized for, variations of the cortical thickness $(t)$ and implant diameter $(d)$, and thus of the $t / d$ ratio, will not necessarily lead to uninterpretable results.

In some situations, a uniform interface stress distribution was found, however, in most situations this stress distribution was not uniform. This is illustrated in Figure 6, where the stress patterns of both a reference situation (equal stress distribution) and an extreme situation (unequal stress distribution) are shown. Both situations can occur in practice, and the resulting stress patterns show the problems that will exist when comparing the two situa- 
tions. It is questioned what the consequences are of a nonuniform stress distribution. A stress peak along the interface represents the area where during the actual push-out test the interface will initially break. Calculation of the shear stress in such a situation by simply dividing the push-out force by the contact area will not give a reliable representation of the true interfacial shear stress. This means that the calculated "stress" can only be regarded as a number that is specific for the test circumstances. This number is hard to compare with data obtained from other situations with a different interface stress distribution. Therefore, the authors state that such comparisons will only give rise to more confusion in interpreting and comparing push-out results. The only comparisons that are meaningful in a situation of nonuniform stress distribution are within the specific standardized experiment at different follow-up periods. It is obvious that in such a situation comparisons of a specific material with others has to rely on other evaluations like histology or electron microscopy as well.

There are several limitations in the present investigation. Our model represents a static situation under a given load, well below the stress that is necessary for fracture. We did not investigate the fracture mechanics of the interface by the push-out test. Furthermore, bone is not isotropic or linear elastic, ${ }^{22}$ and these factors can influence the interface stress distribution. For instance the variability in Young's modulus of the bone surrounding the implant is a relevant parameter, and this will be the topic of a future investigation. We assumed that there was a situation of $100 \%$ bone-bonding, and no fibrous tissue interlayer, which are factors that can vary and thus influence the interface stress pattern. We ignored possible upgrowth of endosteal (trabecular) bone in the model, which for practical situations means that upgrowth is not existing or has been removed. ${ }^{1,11}$ Another factor of influence is the surface characterization of the implant material. A rough surface with a high Young's modulus will give stress peaks at microlevel that can influence especially the fracture mechanics. Implant fixation can be a result of bonding with bone or of mechanical interlocking. Our model addresses the situation of bone bonding. If implant fixation is a result of mechanical interlocking, this model is only suitable up to a certain scale of surface texture (roughness). Of course, when the scale of the surface texture attains a large value, the push-out test loses its basic aspects of measuring plain shear stresses, and becomes a test of a construction.

\section{CONCLUSIONS}

According to the evaluation of the push-out model by finite element analysis, variation of the clearance of the hole in the support jig $(x)$ and Young's modulus of the implant $(E)$ results in different stress distributions along the interface. Therefore these variations lead to uninterpretable test results. In order to optimize the push-out test and to avoid peak stresses, the clearance of the hole in the support jig should be at least $0.7 \mathrm{~mm}$. Materials with different Young's moduli should not be compared with each other in push-out 
tests. Although it is possible to optimize the conditions of a push-out test, the results of such a test always need to be interpreted with the typical uncertainties of a biological system in mind.

This work was financially supported by the Netherlands Technology Foundation (STW).

\section{References}

1. S. D. Cook, K. A. Thomas, J. F. Kay, and M. Jarcho, "Hydroxyapatitecoated porous titanium for use as an Orthopaedic biologic attachment system," Clin. Orthop., 230, 303-312 (1988).

2. S. D. Cook, K. A. Thomas, J. F. Kay, and M. Jarcho, "Hydroxyapatitecoated titanium for orthopaedic implant applications," Clin. Orthop., 232, 225-243 (1988).

3. W. J. A. Dhert, C. P. A.T. Klein, J.G.C. Wolke, E. A. van der Velde, K. de Groot, and P.M. Rozing, "A mechanical investigation of fluorapatite, magnesiumwhitlockite and hydroxylapatite plasma-sprayed coatings in goats," J. Biomed. Mater. Res., in press.

4. W. J.A. Dhert, C.P.A.T. Klein, K. de Groot, J.G.C. Wolke, and P.M. Rozing, "Fluorapatite, magnesiumwhitlockite and hydroxylapatite plasma sprayed coatings on titanium plugs. Push-out tests and the effect of different implantation sites," Trans. Soc. Biomater., 16, 279 (1990).

5. P. Ducheyne, L. L. Hench, A. Kagan, M. Martens, and J.C. Mulier, "Short term bonding behavior of bioglass coatings on metal substrate," Arch. Orthop. Trauma. Surg., 94, 155-160 (1979).

6. T. Fujiu and M. Ogino, "Difference of bond behavior among surface active glasses and sintered apatite," J. Biomed. Mater. Res., 18, 845-859 (1984).

7. R.G.T. Geesink, K. de Groot, and C.P.A.T. Klein, "Chemical implant fixation using hydroxyl-apatite coatings," Clin. Orthop., 225, 147-170 (1987).

8. R.G.T. Geesink, "Hydroxyl-Apatite Coated Hip Implants," Thesis, University of Maastricht, The Netherlands (1988).

9. C. P. A.T. Klein, P. Patka, H. B. M. van der Lubbe, J.G.C. Wolke, and K. de Groot, "Plasma-sprayed coatings of tetracalciumphosphate, hydroxylapatite and alpha-TCP on titanium alloy. An interface study," $J$. Biomed. Mater. Res., 25, 53-65 (1991).

10. R. D. Poser, F. P. Magee, J. F. Kay, and A. K. Hedley, "In vivo characterization of a hydroxylapatite coating," Trans. Soc. Biomater., 16, 170 (1990).

11. K. A. Thomas, S. D. Cook, E. A. Renz, R.C. Anderson, R. J. Haddad, A. D. Haubold, and R. Yapp, "The effect of surface treatments on the interface mechanics of LTI pyroly tic carbon implants," J. Biomed. Mater. Res., 19, 145-159 (1985).

12. K. A. Thomas, J.F. Kay, S. D. Cook, and M. Jarcho, "The effect of surface macrotexture and hydroxylapatite coating on the mechanical strengths and histologic profiles of titanium implant materials," J. Biomed. Mater. Res., 21, 1395-1414 (1987).

13. M. Uratsuji, D. Gaisser, T.W. Bauer, and S.I. Reger, "Bone response to the surface properties of intramedullary rods," Transactions of the $3 r d$ World Biomaterials Congress, 305 (1988).

14. C.C.P. M. Verheyen, W. J. A. Dhert, L. H. Braak, and K. de Groot, "Push-out tests evaluated by finite element analysis," Trans. Soc. Biomater., 17, 216 (1991). 
15. P.S. Boone, M.C. Zimmerman, E. Gutteling, C. K. Lee, J.R. Parsons, and N. Langrana, "Bone attachment to hydroxylapatite coated polymers," J. Biomed. Mater. Res., 23(A2), 183-199 (1989).

16. C. P. A.T. Klein, unpublished data (1991).

17. C.C. P. M. Verheyen, unpublished data (1991).

18. J. Black, "Push-out tests," J. Biomed. Mater. Res., 23, 1243-1245 (1989).

19. K. de Groot, "Letter to the editor," J. Biomed. Mater. Res., 23, 1367-1371 (1989).

20. K.A. Thomas, "Letter to the editor," J. Biomed. Mater. Res., 23, 13671371 (1989).

21. A. Shiradzi-Adl, "Biomechanics of a pull-out test: Evaluation of the interface stresses," Trans. Eur. Soc. Biomech., 35 (1990).

22. M. Nordin and V.H. Frankel, "Biomechanics of whole bones and bone tissue," in Basic Biomechanics of the Skeletal System, V. H. Frankel, and M. Nordin (eds.), Lea and Febiger, Philadelphia, 1980, pp. 15-60.

Received March 3, 1991

Accepted July 25, 1991 